\title{
A significant mass density increase during a large magnetic storm in October 2003 obtained by ground-based ULF observations at $L \sim 1.4$
}

\author{
Satoko Takasaki ${ }^{1,2}$, Hideaki Kawano ${ }^{1}$, Yoshimasa Tanaka ${ }^{3}$, Akimasa Yoshikawa ${ }^{1}$, \\ Masahiro Seto ${ }^{4}$, Masahide Iizima ${ }^{5}$, Yuki Obana ${ }^{6}$, Natsuo Sato ${ }^{2}$, and Kiyohumi Yumoto ${ }^{1,7}$ \\ ${ }^{1}$ Department of Earth and Planetary Sciences, Kyushu University, Fukuoka 812-8581, Japan \\ ${ }^{2}$ now at: Space and Upper Atmospheric Science, National Institute of Polar Research, Tokyo 184-8795, Japan \\ ${ }^{3}$ National Institute of Information and Communications Technology, Tokyo 184-8795, Japan \\ ${ }^{4}$ Tohoku Institute of Technology, Sendai 982-8577, Japan \\ ${ }^{5}$ Graduate School of Science, Tohoku University, Sendai 980-8578, Japan \\ ${ }^{6}$ Venture Business Laboratory, Kyushu University, Fukuoka 812-8581, Japan \\ ${ }^{7}$ Space Environment Research Center, Kyushu University, Fukuoka 812-8581, Japan
}

(Received July 19, 2005; Revised December 1, 2005; Accepted December 1, 2005; Online published May 12, 2006)

\begin{abstract}
During 28-31 October, 2003, a series of coronal mass ejections hit the magnetosphere and triggered two consecutive large storms. Three ground magnetometers at $L=1.32-1.41$ recorded field-line resonances (FLRs) during this interval. The FLR frequencies decreased from 0600 LT on 31 October 2003 during the main phase of the second storm until 12 LT when the recovery phase of this storm began. After the decrease, the FLR frequencies returned to its pre-storm value (at $0600 \mathrm{LT}$ on 31 Oct.) in a few hours. The measured decrease in the FLR frequency suggests a relative increase in mass density along the field lines during the magnetic storm. On the other hand, the total electron content (TEC) data suggest that the ionospheric plasma number density during this storm was similar to that during quiet times. A possible explanation for the increase in mass density would be an outflow of the heavy ions (e.g., $\mathrm{O}^{+}$) from the ionosphere to the plasmasphere.
\end{abstract}

Key words: Plasmasphere, magnetic storms, remote sensing, ionosphere/magnetosphere interactions.

\section{Introduction}

It is well known that hydromagnetic waves are observed everywhere in the Earth's magnetosphere. The field-line resonance (FLR) is excited when the frequency of hydromagnetic waves in the magnetosphere matches the field-line eigenfrequency (Orr, 1984; Allan and Poulter, 1992). As a rough approximation, FLRs are considered as the oscillation to propagate with shear Alfvén waves traveling along the magnetic field lines between the northern and southern ionospheric boundary (Obayashi and Jacobs, 1958). The Alfvén speed $\left(V_{A}\right)$ is defined as $V_{A}=(4 \pi \rho)^{-1 / 2} B$, where $\rho$ is the plasma mass density and $B$ is the magnetic field intensity. Thus the field-line eigenfrequency (i.e. the FLR frequency) is expected to be a function of the field-line length, the magnetic field intensity, and the mass density along the field line (Kitamura and Jacobs, 1968; Troitskaya, 1997).

At low geomagnetic latitudes, geomagnetic field lines are considered to be stable. Thus FLR frequency observed at a fixed point shows inverse proportion to the plasma mass density along field lines. As FLR mainly occurs in the dayside, a network observation of ground magnetometers is useful for continuously monitoring the FLR frequency, from which we can estimate qualitative changes of the plasma mass density in the inner plasmasphere. Aiming to investigate the plasma mass density in the inner plasma-

Copyright (c) The Society of Geomagnetism and Earth, Planetary and Space Sciences (SGEPSS); The Seismological Society of Japan; The Volcanological Society of Japan; The Geodetic Society of Japan; The Japanese Society for Planetary Sciences; TERRAPUB. sphere, we have installed two low latitude ground magnetometers located at $L=1.32$ and 1.41 in 2003 and succeeded in monitoring the FLR frequency. There are no previous reports on the changes in FLR frequency during large magnetic storms at the low-latitude region $L \sim 1.4$, so that our study in this report would be important.

This paper constitutes the first report of a significant decrease in the FLR frequency at $L=1.32-1.41$ during a large magnetic storm. The decrease in the FLR frequency took place during the second of two consecutive large storms on 31 October 2003. It is certain that this decrease in the FLR frequency was caused by the increase in the mass density along the field line at $L=1.32-1.41$, because, as will be stated later, the field lines there are hardly deformed even during magnetic storms. We consider a possible explanation for this mass-density increase.

Some previous literature, which are the studies at higher latitudes than our study in this paper, reported the plasma mass density inferred from observed FLR frequency (e.g. Chi et al., 2000; Chi, 2005; Vellante et al., 2002; Clilverd et al., 2003; Menk et al., 1999, 2004). Menk et al. (2004) studied the latitudinal profiles of mass density at $2.5<L<10$ and investigated the position of the plasmapause under quiet and disturbed (but non-storm) conditions. Furthermore, they discussed the heavy ion mass loading effect to the field lines by comparing the mass density inferred from the FLR frequency with the electron number density determined by using natural VLF whistlers. Clilverd et al. (2003) also compared the mass density inferred from the 
FLR frequency with ground-based whistler and in-situ observed electron density data at $L=2.5$ during relatively quiet condition. During the main phase of magnetic storms, temporal variations in the mass density inferred from FLR frequency were reported by Chi et al. (2000), Chi (2005) and Vellante et al. (2002) at $L=1.55 \sim 3$.

It is noted that Chi (2005) reported an increase in the mass density at higher latitudes $(L=2.5 \sim 3.0)$ during the same storm event as this study. The density maximum observed by Chi occurred at least 5-hour before the maximum reported in this paper. In addition to that, these storm-time increase is in contrast with previously-reported storm-time density decreases at $L=1.55 \sim 2$, reported for different smaller storms (Chi et al., 2000; Vellante et al., 2002).

We also document the ionospheric response during the same time interval using total electron content (TEC) data deduced from GPS signals, and study the contribution of ions outflowing from the ionosphere to the plasmasphere.

The observations presented in this paper, made at $L<1.4$, could be combined in the future with the observations at higher $L$, which would contribute to understanding the behaviors of the global variation of the plasmaspheric mass density in storm time.

\section{Data Analysis}

Magnetometers were installed at Tohno (TNO, geographic coordinates: $\left.39.37^{\circ} \mathrm{N}, 141.60^{\circ} \mathrm{E}\right)$ in 2003 , and at Iitate (ITA, geographic coordinates: $37.70^{\circ} \mathrm{N}, 140.34^{\circ} \mathrm{E}$ ) in 2000 by Kyushu University, Tohoku Institute of Technology, and Tohoku University. The fluxgate magnetometers at these stations record the geomagnetic $H, D$, and $Z$ components with a resolution of $0.01 \mathrm{nT}$, and the sampling time is $1 \mathrm{sec}$ with precise GPS timing receiver. The magnetic latitudes ( $L$-values) of TNO and ITA are $32.48^{\circ}(1.41)$ and $30.90^{\circ}(1.36)$, respectively, and both stations are located approximately along the $210^{\circ}$ magnetic meridian.

The 'gradient methods' (Baransky et al., 1989; Waters et al., 1991; Kawano et al., 2002) are designed to extract FLR from ground magnetometer data using two stations closely spaced along the same meridian. In this paper, we distinguished FLRs by applying the gradient methods to the storm-time data using ground magnetometers at $L \sim 1.4$. Our methods are briefly reviewed below.

The cross-phase technique and the amplitude-ratio technique are used to identify the resonance frequency of a point between the two stations. The concept of the crossphase technique (as named by Waters et al. (1991)) was first proposed by Baransky et al. (1989). The phase of the $H$-component jumps by 180 degrees across the resonance point (Baransky et al., 1985). The latitudinal gradient of the phase is steepest at the resonance point; the phase difference of the $H$-component between the two stations is therefore expected to be largest when the resonance point is located at a point between the two stations. We can therefore determine the peak frequency to be the resonance frequency at a point between the two stations. The amplitude of the $H$ component magnetic pulsations reaches a maximum at the resonance point (Baransky et al., 1985). The ratio of the dynamic spectra of the $H$-component data at two ground stations of contrasting latitude will produce a bipolar structure because the amplitude response of the resonance at the two sites is maximized at different frequencies. We can therefore determine the frequency at the center of the bipolar signature to be the resonance frequency at a point between the two stations.

The two storms in this study took place during 29-31 October 2003. Figure 1 shows the results of the gradient methods applied to the data of 28,29 , and 31 October, when FLRs appeared. As FLRs occur mainly in the dayside, all spectrograms present the interval of 2000 UT to $0900 \mathrm{UT}$, which corresponds to the local time (LT) of $0500 \mathrm{LT}$ to $1800 \mathrm{LT}$. The white color in this figure corresponds to low coherence less than 0.7 .

The clear ridge (red color) across each top panel in Fig. 1 indicates the cross-phase maximum. The ridge corresponds to the maximum value of the phase difference from ITA to TNO, phase difference is positive in this case. The reason of the positive value of the phase difference is as follows. The field-line eigenfrequency and the phase of the $H$-component generally increase with increasing latitude at $L<1.6$ (Menk et al., 2000) due to ionospheric $\mathrm{O}^{+}$mass loading to flux tubes at $L<1.6$ (Hattingh and Sutcliffe, 1987; Poulter et al., 1988). Subtraction of the phase at ITA from that at TNO (located at a higher latitude) therefore results in a positive value.

The latitude dependence of the FLR frequency outlined above can also be identified from the amplitude-ratio pattern in Fig. 1: There is a clear trough (blue color) and a clear ridge (red) across each of the lower panels (the trough is at a lower frequency than the ridge). The trough represents less power at TNO than at ITA, while the ridge represents greater power at ITA. We can therefore identify the trough as a resonance over the ITA station and the ridge as a resonance over the TNO station. The observed pattern indicates that the FLR frequency increased with increasing geomagnetic latitude.

On 28 October the FLR only appeared over a very short interval (Fig. 1(a)). Starting from 1300 LT, the eigenfrequency of the magnetospheric field line at a point between TNO and ITA can be clearly identified at $70 \mathrm{mHz}$ in the two panels of Fig. 1(a); the eigenfrequency decreased slightly from 1300 LT to $1440 \mathrm{LT}$.

On 29 October the first magnetic storm started at 1511 LT. On this day, FLR (Fig. 1(b)) was not as clear as FLR represented in Figs. 1(a) and 1(c). FLR at 0820 LT had a frequency of $75-85 \mathrm{mHz}$. FLR had a frequency of $65-70 \mathrm{mHz}$ at $1420 \mathrm{LT}$ when a sudden commencement was observed at the stations. From 1500-1620 LT, phase difference and amplitude ratio signatures were observed at high frequencies $(90-120 \mathrm{mHz})$, which corresponds to higher FLR harmonics. The fundamental mode is identified at 50 $65 \mathrm{mHz}$.

The second magnetic storm began at 0137 LT on 31 October 2003. On this day, FLR was clearly identified during 0640-1200 LT and 1350-1600 LT (Fig. 1(c)). The observed eigenfrequency steadily decreased from $55 \mathrm{mHz}$ at $0640 \mathrm{LT}$ to $40 \mathrm{mHz}$ at $1200 \mathrm{LT}$. FLR appeared again at $1350 \mathrm{LT}$, and the eigenfrequency increased from $60 \mathrm{mHz}$ to $65 \mathrm{mHz}$ during the period 1350-1600 LT. Higher harmonics of FLR, at about $90 \mathrm{mHz}$, were observed during 
(a) Oct. 28, 2003

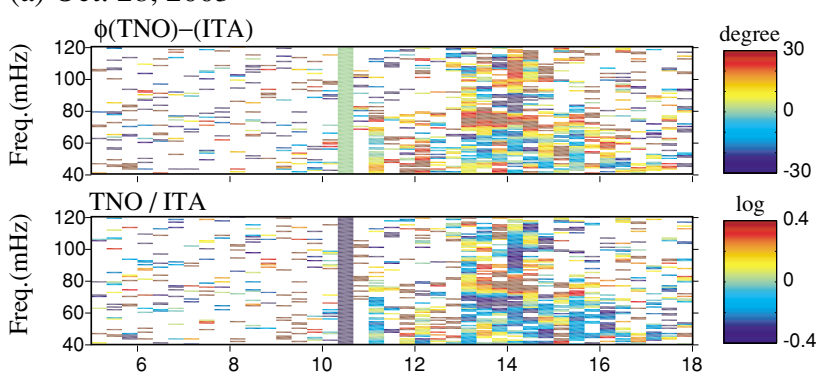

(b) Oct. 29, 2003

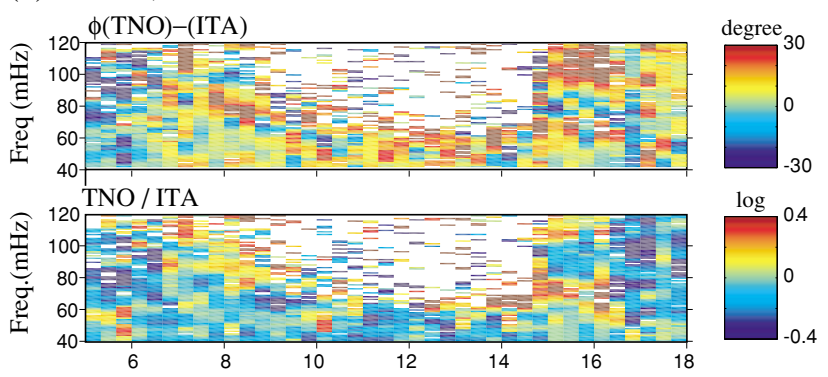

(c) Oct. 31, 2003

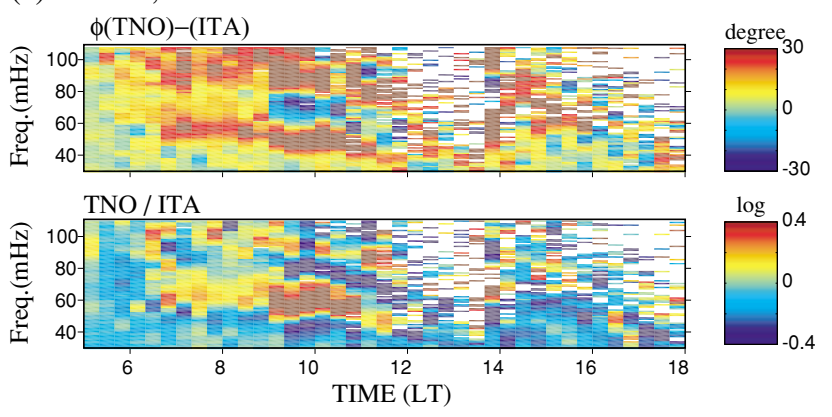

Fig. 1. The upper figures within Figs. 1(a), 1(b), and 1(c) show the phase difference of the $H$-component spectra from ITA to TNO. The lower figures within Figs. 1(a), 1(b), and 1(c) show the ratio of the $H$-component spectra at TNO to the spectra at ITA. Each figure shows the spectrogram for the local time interval 0500-1800 LT. The first magnetic storm started at 0611 UT (1511 LT) on 29 October and the second magnetic storm started at 1637 UT (0137 LT on the folowing day) on 30 October. As FLRs only occur on the dayside, all spectrograms present the interval from 2000 UT to 0900 UT of the following day, which corresponds to the local time interval from $0500 \mathrm{LT}$ to $1800 \mathrm{LT}$. The white color corresponds to low coherence less than 0.7 .

\section{0-1200 LT.}

We also made the same analysis the station pair ITAKAK, where KAK is Kakioka $(L=1.32$, [magnetic lat., magnetic long.] $=[29.39,211.89])$, and obtained almost identical results. These results will be discussed in the next section.

\section{Results and Discussion}

The FLR frequencies shown in Fig. 2 were determined as follows. The ground magnetometer data were divided into 20-minute intervals. For each interval, we used the crossphase and amplitude-ratio methods to determine if FLR exists in the $H$-component data from the TNO and ITA stations. If both methods identified FLR within a given interval, we examined if the two values of the FLR frequencies derived from the two methods were in agreement (allowing for a $5 \mathrm{mHz}$ difference between the two values). If the val-

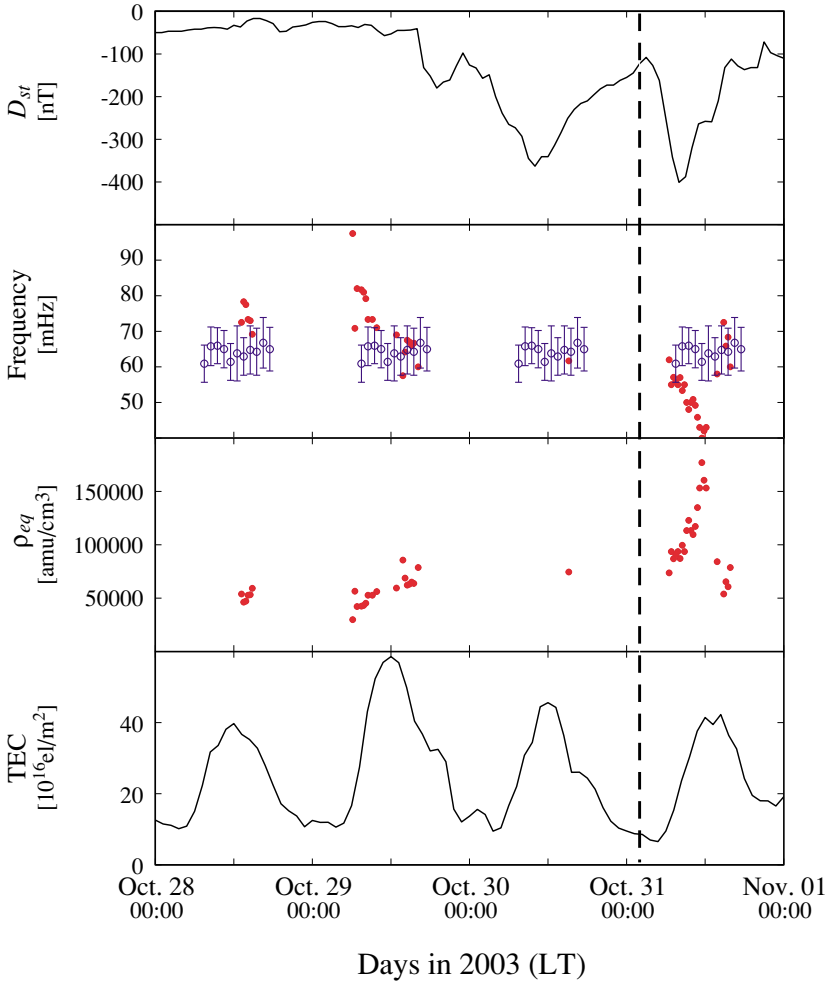

Fig. 2. (From top to bottom) $D_{s t}$ index, field-line resonant frequencies derived from ground observations, $\rho_{\mathrm{eq}}$ estimated from data in the second panel, and the total electron content (TEC). The dashed vertical line marks the beginning of the second magnetic storm. The second panel shows the mean FLR frequency (blue open circles) and the standard deviation (blue errorbars) in the quiet times. The red filled circles in the second panel correspond to the FLR frequencies actually observed during the interval of the figure.

ues were in good agreement, we concluded that FLR existed during the 20-min interval, and record the eigenfrequency.

Time series data of thus identified FLR frequencies are shown in the second panel of Fig. 2 as filled red circles in the same time frame as the $D_{s t}$ index (top panel). The time interval displayed in Fig. 2 covers the two storms described in this paper.

We have also studied the diurnal variations of the FLR frequency during quiet times, and examined whether the variations during the second storm on Oct. 31 were unusual. For all the quiet-time datapoints during May 23, 2000Dec. 31, 2003, the criteria for the quietness are as follows: $K_{p}<3, D_{s t}<40 \mathrm{nT}$, IMF $B_{\text {total }}<10 \mathrm{nT}$, and the solar wind dynamic pressure $P_{\text {dyn }}<5 \mathrm{nPa}$. The solar wind parameters used in this analysis are obtained by the ACE satellite (Smith et al., 1998; McComas et al., 1998). For IMF $B_{\text {total }}$ and $P_{\text {dyn }}$, we have roughly corrected the solar wind propagation lag by dividing the distance between the Earth and ACE by the solar wind bulk speed.

The second panel of Fig. 2 shows the mean FLR frequency (blue open circles) and the standard deviations (blue errorbars) in the quiet times as a function of LT. It is clearly seen that the FLR frequeny during the second storm significantly decreased from the mean diurnal variation of FLR frequency in quiet times. On the other hand, on 29 October, the FLR frequency at 0600 LT was higher than the mean 
value and decreased to the mean value till the noon, before the onset of the first storm.

This is the first study to describe a significant decrease in FLR frequency during a large magnetic storm at lowlatitudes $(L=1.32-1.41)$. We believe that the decrease in FLR frequency recorded on 31 October was causally related to the second storm, because the FLR frequency at $L=1.32-1.41$ also decreased in most other large storms that we examined (not shown here).

As documented in Introduction, the FLR frequency is affected by the field-line length, the magnetic field intensity, and the mass density along the field line. If the field-line is only slightly distorted during magnetic storms, the decrease of the FLR frequency is mainly caused by an increase in plasma mass density along the magnetic field line.

Here we estimate how much field lines were distorted during the storm events. In this study, the magnetic field intensities $\left(|B|_{\text {eq }}\right)$ in the equatorial region and the lengths of the field line $(l)$, under quiet and disturbed conditions have been calculated using the most recent model of Tsyganenko and Sitnov (2005). We have calculated the model magnetic field at $1100 \mathrm{LT}$ at TNO located at $L \sim 1.4$ on 30 Oct., 2003, with the following two sets of model parameters corresponding to the quiet and disturbed conditions: Solar wind dynamic pressure $P_{\text {dyn }}: 3 \mathrm{nPa}$, IMF $B_{y}: 3 \mathrm{nT}$, IMF $B_{z}: 3 \mathrm{nT}$ and $D_{s t}$ index: $15 \mathrm{nT}$ as the quiet condition, and $P_{\text {dyn }}: 20 \mathrm{nPa}$, IMF $B_{y}:-20 \mathrm{nT}$, IMF $B_{z}:-20 \mathrm{nT}$ and $D_{s t}$ index: $-300 \mathrm{nT}$ as the disturbed condition. These disturbed-condition values were actually observed by the ACE satellite when the FLR frequency was minimized during the second storm of this paper.

As a result of the calculation, the equatorial $|B|_{\mathrm{eq}}$ and $l$ under the disturbed condition differs by only $0.2 \%$ and $0.08 \%$, respectively, from that under the quiet condition. Hence, it is certain that the variation in the FLR frequency for our event was mainly caused by the change of the mass density along the filed line. Thus, in the following we will estimate the qualitative change in mass density along the field line from the recorded decreases in FLR frequency.

The primary aim of this paper is to determine and interpret variation in mass density along the field-line rather than to obtain precise quantitative estimates of the mass density. Therefore, in the following text, we will concentrate on whether the mass density increased or decreased during the storm event.

We use the method of Schulz (1996) to estimate $\rho_{\text {eq }}$ from the field-line eigenfrequency. We assume a dipole magnetic field and a power-low plasma mass density model $\rho=\rho_{\mathrm{eq}}(L / r)^{m}$, where $\rho$ is the plasma mass density, $L$ is the $L$-value of the field line, and $r$ is the geocentric distance (in $R_{E}$ ) of the point of interest upon the field line. We assign parameter $m$ of the plasma mass model a value of four, consistent with previous studies (Chi et al., 2000).

The procedure suggested by Schulz (1996) does not provide a quantitatively precise $\rho_{\text {eq }}$ estimate for low-latitude field lines (at $L<1.8$ ) because a significant portion of the field lines lie within the ionosphere. The mass loading effect of the ionospheric heavy ions steepens the plasma density gradient along the magnetic field line and this effect act to lower the eigenfrequency. However, as stated above, we pay attention only to the general trend of variation in $\rho_{\mathrm{eq}}$; for such qualitative information, the procedure of Schulz is adequate even at low latitudes. Procedures for determining more precise estimates can be found in the literature (e.g. Hattingh and Sutcliffe, 1987; Poulter et al., 1988; Price et al., 1999).

The third panel of Fig. 2 shows the density estimated via the method of Schulz. The density increased before the first storm, on Oct. 29, and again during the second storm on Oct. 31. It is found that the second increase was significantly larger than the first. The discontinuous nature of the data makes it impossible to determine if the density changed during the first storm.

The fourth panel of Fig. 2 shows TEC values measured on the ground at $L=1.38$ ([magnetic lat., magnetic long. $]=[31.18,212.48])$. The TEC values were measured by the Geographical Survey Institute (GSI) GPS network, GEONET (GPS Earth Observation Network). TEC data are obtained every 30 seconds for all of the satellite-receiver paths of GEONET (Saito et al., 1998). We used the method of Otsuka et al. (2002) to remove instrumental biases inherent in the GPS satellite and receiver, and then we determined absolute values of TEC. The panel shows vertical TEC, estimated from the TEC of many different paths measured at each station.

A significant increase in the daily TEC maximum occurred on 29 October before the main phase of the first magnetic storm. This increase was simultaneous with the increase in $\rho_{\mathrm{eq}}$ at $L \sim 1.4$ (deduced from our ground magnetometer data) while $D_{s t}$ was relatively constant immediately prior to the storm on 29 October. It is therefore likely that the increases in TEC and $\rho_{\mathrm{eq}}$ are related phenomena. A possible explanation for this relationship is that plasma outflew from the ionosphere, which had a higher number density than usual (as suggested by TEC), toward the plasmasphere at $L \sim 1.4$ and resulted in an increase in mass density at this point. In any case, the density increase preceding the first storm was smaller in magnitude than that following the onset of the second storm.

The significant decrease in the FLR frequency during the second magnetic storm is explained by the increase of the mass density along the field line; the source of the increased mass density along the field line at $L \sim 1.4$ might be the ionospheric plasma outflow, which is an ionospheric-storm effect.

However, the daily TEC maximum during the main phase of the second magnetic storm was similar in magnitude with that during quiet time (e.g., 28 October). This observation indicates that the relative plasma number density in the ionosphere did not increase significantly, because the majority of TEC comes from the ionosphere. Outflow of the ionospheric plasma was therefore minimal, even though the equatorial plasmaspheric mass density estimated from FLR significantly increased over the same time interval (Fig. 2, third panel). Despite these observations, the ionosphere remains the most likely source of the increased mass, as discussed in the following.

During the second storm a small amount of the ionospheric plasma outflew, causing little change in TEC. However, the small increase in the plasmaspheric number den- 
Table 1. The result of the model calculation of the equatorial magnetic intensity $|B|_{\text {eq }}$ and the field-line length $l$ under the quiet condition and the strongly disturbed condition.

\begin{tabular}{ccccccc}
\hline & $\begin{array}{c}B_{y} \\
{[\mathrm{nT}]}\end{array}$ & $\begin{array}{c}B_{z} \\
{[\mathrm{nT}]}\end{array}$ & $\begin{array}{c}P_{\mathrm{dyn}} \\
{[\mathrm{nPa}]}\end{array}$ & $\begin{array}{c}D_{s t} \\
{[\mathrm{nT}]}\end{array}$ & $\begin{array}{c}|B|_{\mathrm{eq}} \\
{[\mathrm{km}]}\end{array}$ & $\begin{array}{c}l \\
{[\mathrm{nT}]}\end{array}$ \\
\hline quiet time & 3 & 3 & 3 & 15 & 12553 & 10666 \\
storm time & -20 & -20 & 20 & -300 & 12583 & 10676 \\
\hline
\end{tabular}

sity corresponded to a large increase in the mass density as shown by our FLR data. Such features would be caused by heavy ions (e.g., $\mathrm{O}^{+}$), which are the main constituent of the ionospheric plasma outflow (Kelley, 1989), so that the resultant small increase in the plasmaspheric number density meant a lot of increase in the mass density there (as shown in our FLR data).

We also note that the increased mass could return to its pre-storm value within a few hours after the storm onset because the Earth's gravity makes heavy ions sink into the ionosphere faster than $\mathrm{H}^{+}$; this could explain the observed density decrease in a few hours after the density maximum at $\sim 12 \mathrm{hr}$ LT on 31 Oct. (Fig. 2, third panel).

Chi et al. (2000) and Vellante et al. (2002) reported a decrease in the plasmaspheric mass density at $L=1.55-2$ during the main phases of magnetic storms. The authors concluded that the decrease resulted from depletion of the plasmasphere. On the other hand, Förster et al. (1992) reported that the magnitude of the $\mathrm{O}^{+}$densities increased during a magnetic storm with decreasing latitude. Applying for their results to our event at $L=1.32-1.41$, we can conjecture that the $\mathrm{O}^{+}$enhancement may have overcome the density-decrease effect, because the $\mathrm{O}^{+}$enhancement would have been more effective during the event in this study than during the other storms, which Chi et al. (2000) and Vellante et al. (2002) studied. In addition, there is the possibility that the enhancement of the $\mathrm{O}^{+}$density as reported by Förster et al. is more significant (at all latitudes) for larger storms.

Chi (2005) reported an increase similar to the increase in our paper, but at larger $L(L=2.5 \sim 3)$ and at different local time ( $\sim 8.5$ hours to the east of our stations), during the same second storm. The density maximum observed by Chi occurred at 21 UT (14 LT) on 30 October, and the density maximum reported in this paper occurred at 3 UT (12 LT) on 31 October. In other words, the density maximum in our paper occurred at least five hours after the density maximum of Chi (2005).

Chi suggested that the eastward electric field uplifted the ionospheric F-layer. On the other hand, the maximum of the mass density reported in this paper occurred at least 5-hour after the maximum observed by Chi, when the electric field was westward (as shown in figure 3 of Chi (2005)). Thus, we suspect that, at $L \sim 1.4$, when the electric field does not directly influence the significant increase in the mass density along the field line. The increase in the mass density could be due to the uplifted ionosphere along the field line by the enhanced equatorward neutral wind in the thermosphere generated by the neutral-gas heating in the auroral zone during the main phase of the storm. Chi referred to
Takasaki et al. (2004) and stated that further studies, which combine observations at different local times would be very useful in understanding how relevant physical mechanisms influence the behaviors of the storm-time plasmashpereionosphere system. We take the same view as them, and we also note that it is also important to combine observations at diffferent $L$ 's.

\section{Summary}

This paper shows a significant decrease in the FLR frequency at $L \sim 1.4$ during the large magnetic storm. There are some possible explanations for the decrease; the FLR frequency decrease when the magnetic field-line becomes distorted, and/or the mass density along the field line increases. We calculated the paths of the field line by the latest Tsyganenko model (Tsyganenko and Sitnov, 2005) and found that the magnetic field-line at low latitude $(L \sim 1.4)$ is rarely distorted even in large magnetic storm. Therefore, we mainly considered the most possible explanation that the decrease might have resulted from an increase in the mass density along field line. On the other hand, TEC data suggest that the ionospheric plasma number density during this storm was similar to that during quiet times. These different responses between the mass density estimated from the FLR frequency and the ionospheric number density inferred from the TEC values indicate that a small outflow of the heavy ions (e.g., $\mathrm{O}^{+}$) from the ionosphere increased the mass density in the plasmasphere.

Acknowledgments. The $D_{s t}$ index was provided by the World Data Center for Geomagnetism. Geomagnetic data from Kakioka were provided by the Kakioka Magnetic Observatory, Japan. We acknowledge the Geographical Survey Institute (GSI), Japan for providing the GEONET data. This study has been supported by the Professor Tatsuro Matsumoto Scholarship Fund and Grant-inAids 12373003 and 13554016 of MESSC.

\section{References}

Allan, W. and E. M. Poulter, ULF waves - their relationship to the structure of the earths magnetosphere, Rep. Prog. Phys., 55, 533, 1992.

Baransky, L. N., Y. E. Borovkov, M. B. Gokhberg, S. M. Krylov, and V. A. Troitsukaya, High resolution method of direct measurement of the magnetic field line's eigenfrequencies, Planet. Space Sci., 36, 13691375, 1985.

Baransky, L. N., S. P. Belokris, Y. E. Borovkov, M. B. Gokhberg, E. N. Fedorov, and C. A. Green, Restoration of the meridional structure of geomagnetic pulsation field from gradient measurement, Planet. Space Sci., 37, 859-864, 1989.

Chi, P. J., Density enhancement in plasmasphere-ionosphere plasma during the 2003 halloween superstorm: Observations along the 330th magnetic meridian in North America, Geophys. Res. Lett., 32, L03S07, doi:10.1029/2004GL021722, 2005.

Chi, P. J., C. T. Russell, S. Musman, W. K. Peterson, G. Le, V. Angelopoulos, G. D. Reeves, M. B. Moldwin, and F. K. Chun, Plasmaspheric and 
refilling associated with the September 25, 1998 magnetic storm observed by ground magnetometers at $L=2$, Geophys. Res. Lett., 27, 633-636, 2000.

Clilverd, M. A., et al., In-situ and ground-based intercalibration measurements of plasma density at $L=2.5$, J. Geophys. Res., 108(A10), 1365, doi:10.1029/2003JA009866, 2003.

Förster, M., N. Jakowski, A. Best, and J. Smilauer, Plasmaspheric response to the geomagnetic storm period March 20-23, 1990, observed by the activity (MAGION-2) satellite, Canadian Journal of Physics, 70(7), 569-574, 1992.

Hattingh, S. K. F. and P. R. Sutcliffe, Pc 3 pulsation eigenperiod determination at low latitudes, J. Geophys. Res., 92, 12,433-12,436, 1987.

Kawano, H., K. Yumoto, V. A. Pilipenko, Y. M. Tanaka, S. Takasaki, M. Iizima, and M. Seto, Using two ground stations to identify magnetospheric field line eigenfrequency as a continuous function of ground latitude, J. Geophys. Res., 107, doi:10.1029/3001JA000274, 2002.

Kelley, M. C. (Ed.), The Earth's Ionosphere: Plasma Physics and Electrodynamics, International Geophysics Series, Academic Press, San Diego, 1989.

Kitamura, T. and J. A. Jacobs, Determination of magnetospheric plasma density by the use of long-period geomagnetic micropulsations, Geomagn. Geoelec., 20, 33, 1968.

McComas, D. J., S. J. Bame, S. J. Barker, W. C. Feldman, J. L. Phillips, P. Riley, and J. W. Griffee, Solar wind electron proton alpha monitor (SWEPAM) for the advanced composition explorer, Space Sci. Rev., 86, 563-612, 1998.

Menk, F. W., D. Orr, M. A. Clilverd, A. J. Smith, C. L. Waters, D. K. Milling, and B. J. Fraser, Monitoring spatial and temporal variations in the dayside plasmapshere using geomagnetic field line resonance, $J$. Geophys. Res., 104(A9), 19,995-19,969, 1999.

Menk, F. W., Waters, and B. J. Fraser, Field line resonances and waveguide mode at low latitudes 1 . Observation, J. Geophys. Res., 105(A4), doi:10.1029/1999JA900268, 2000.

Menk, F. W., I. R. Mann, M. A. C. A. J. Smith, C. K. Waters, and D. J. Milling, Monitoring the plasmapause using geomagnetic field line resonances, J. Geophys. Res., 109(A04216), doi:10.1029/2003JA010097, 2004.

Obayashi, T. and J. A. Jacobs, Geomagnetic pulsations and the earth's outer atmosphere, Geophys. J. R. Astr. Soc., 1, 53, 1958.

Orr, D., Magnetospheric hydromagnetic waves: Their eigenperiods, ampli- tudes and phase variations; a tutorial introduction, J. Geophys. Res., $\mathbf{5 5}$ 1984.

Otsuka, Y., T. Ogawa, A. Saito, T. Tsugawa, S. Fukao, and S. Miyazaki, A new technique for mapping of total electron content using GPS network in japan, Earth Planets Space, 54, 63-70, 2002.

Poulter, E. M., W. Allan, and G. J. Bailey, ULF pulsation eigenperiods within the plasmasphere, Planet. Space. Sci., 36, 185-196, 1988.

Price, I. A., C. K. Waters, F. W. Menk, G. J. Bailey, and B. J. Fraser, A technique to investigate plasma mass density in the topside ionosphere using ulf waves, J. Geophys. Res., 104(A6), 12,723-12,732, 1999.

Saito, A., S. Fukao, and S. Miyazaki, High resolution mapping of tec perturbations with the GSI-GPS network over japan, Geophys. Res. Let., 25, 3079-3082, 1998.

Schulz, M., Eigenfrequencies of geomagnetic lines and implications for plasma-density modeling, J. Geophys. Res., 101, 17,385-17,397, 1996.

Smith, C. W., J. L'Heureux, N. F. Ness, M. H. Acuna, L. F. Burlaga, and J. Scheifele, The ace magnetic field experiment, Space Sci. Rev., 86, 613-632, 1998.

Takasaki, S., H. Kawano, Y. Tanaka, A. Yoshikawa, M. Seto, M. Iizima, and K. Yumoto, Density perturbations in the inner plasmasphere during huge magnetic storms in October-November 2003: Ground-based observations, Eos Trans. AGU, 85(17), Jt. Assem. Suppl., Abstract SH53A11, 2004.

Troitskaya, V. A., Early ground based approach to hydromagnetic diagnostics of outer space, Discovery of the Magnetosphere, History of Geophysics, 7, 221, 1997.

Tsyganenko, N. A. and M. I. Sitnov, Modeling the dynamics of the inner magnetosphere during strong geomagnetic storms, J. Geophys. Res., 110(A03), doi:10.1029/2004JA010798, 2005.

Vellante, M., M. D. Lauretis, M. Förster, S. Lepedi, B. Zieger, U. Villante, V. A. Pilipenko, and B. Zolesi, Geomagnetic field line resonances at low latitudes: pulsation event study of august 16, 1993, J. Geophys. Res., 107(A5), 1-20, 2002.

Waters, C. L., F. W. Menk, and B. J. Fraser, The resonance structure of low latitude Pc 3 geomagnetic pulsations, Geophys. Res. Lett., 18, 17,547, 1991.

S. Takasaki (e-mail: stakasaki@uap.nipr.ac.jp), H. Kawano, Y. Tanaka, A. Yoshikawa, M. Seto, M. Iizima, Y. Obana, N. Sato, and K. Yumoto 